\section{Inheritance of Neural Substrates for Motivation and Pleasure}

\author{
Zhi Li' ${ }^{1,2}$, Yi Wang ${ }^{1}$, Chao Yan ${ }^{3}$, Eric F. C. Cheung ${ }^{4}$, \\ Anna R. Docherty ${ }^{5,6}$, Pak C. Sham ${ }^{7,8,9}$, Raquel E. Gur ${ }^{10}$, \\ Ruben C. Gur ${ }^{10}$, and Raymond C. K. Chan ${ }^{1,2}$ iD \\ ${ }^{1}$ Neuropsychology and Applied Cognitive Neuroscience Laboratory, CAS Key Laboratory of Mental Health, \\ Institute of Psychology, Chinese Academy of Sciences; ${ }^{2}$ Department of Psychology, Chinese Academy \\ of Sciences; ${ }^{3}$ Key Laboratory of Brain Functional Genomics, School of Psychology and Cognitive Science, \\ East China Normal University; ${ }^{4}$ Castle Peak Hospital, Hong Kong, China; ${ }^{5}$ Department of Psychiatry, \\ University of Utah School of Medicine; ${ }^{6}$ Virginia Institute for Psychiatric and Behavioral Genetics, \\ Virginia Commonwealth University; ${ }^{7}$ Centre for Genomic Sciences, The University of Hong Kong; \\ ${ }^{8}$ State Key Laboratory in Brain and Cognitive Sciences, The University of Hong Kong; ${ }^{9}$ Department of \\ Psychiatry, The University of Hong Kong; and ${ }^{10}$ Department of Psychiatry, Perelman School of Medicine, \\ University of Pennsylvania
}

Psychological Science 2019, Vol. 30(8) 1205-1217 (C) The Author(s) 2019 Article reuse guidelines: sagepub.com/journals-permissions DOI: $10.1177 / 0956797619859340$ www.psychologicalscience.org/PS @SAGE

\section{Abstract}

Despite advances in the understanding of the reward system and the role of dopamine in recent decades, the heritability of the underlying neural mechanisms is not known. In the present study, we examined the hemodynamic activation of the nucleus accumbens (NAcc), a key hub of the reward system, in 86 healthy monozygotic twins and 88 healthy dizygotic twins during a monetary-incentive-delay task. The participants also completed self-report measures of pleasure. Using voxelwise heritability mapping, we found that activation of the bilateral NAcc during the anticipation of monetary gains had significant heritability $\left(b^{2}=.20-.49\right)$. Moreover, significant shared genetic covariance was observed between pleasure and NAcc activation during the anticipation of monetary gain. These findings suggest that both NAcc activation and self-reported pleasure may be heritable and that their phenotypic correlation may be partially explained by shared genetic variation.
\end{abstract}

\title{
Keywords
}

reward system, nucleus accumbens, heritability, motivation, pleasure

Received 8/14/18; Revision accepted 5/9/19

The reward system plays a key role in human behavior and emotion (Iversen, 2010). The nucleus accumbens (NAcc), situated in the ventral striatum, is regarded as the hub of the mesolimbic and mesocortical reward systems (Baldo \& Kelley, 2007; Haber \& Knutson, 2010). Compelling evidence supports the notion that reward processing can be parsed into anticipatory and consummatory phases (Baldo \& Kelley, 2007). Dopaminergic activity in the NAcc is associated with salience attribution of motivation, which assigns motivational significance to different incentives in the anticipatory period (Berridge, 2003; Berridge \& Robinson, 1998). Despite these advances in understanding the reward system, whether reward processing and its component phenotypes are heritable is largely unknown.
Studies in the past decade have suggested a relationship between dopaminergic gene variation and ventral striatal activation measured by functional MRI (fMRI) reward tasks in anticipating and predicting monetary incentives (Dreher, Kohn, Kolachana, Weinberger, \& Berman, 2009; Forbes et al., 2009; Yacubian et al., 2007). Although single-nucleotide polymorphisms such as the dopamine transporter and catechol-O-methyltransferase genes have been associated with ventral striatal activation to a modest extent (Forbes et al.,

\section{Corresponding Author:}

Raymond C. K. Chan, Chinese Academy of Sciences, Institute of Psychology, 16 Lincui Rd., Room 526, South Block, Beijing 100101, China E-mail: rckchan@psych.ac.cn 
2009), studies that examined single polymorphisms have seldom been replicated and tend to be underpowered. Evidence from the Psychiatric Genomics Consortium indicates that psychiatric phenotypes are polygenic, with any one gene contributing only a small amount to the overall variance. Thus, it is essential either to use genome-wide common-variant data, aggregating the effects of all polymorphisms, or to examine familybased genetic variation to have meaningful explanatory power. The prediction of ventral striatal activation using polygenic risk scores for psychosis has already revealed the cumulative effect of genes (Bossong \& Kahn, 2016), and it is expected that related component phenotypes are similarly polygenic. However, the extent to which genetic factors influence NAcc and ventral striatal activation, particularly in the anticipatory period for reward, is not clearly understood.

In this study, we examined measures of shared genetic variance among dimensional psychiatric phenotypes using a voxelwise topographical approach to map striatal activation. Whereas region-of-interest (ROI) methodology has mainly been employed in previous studies, voxelwise analysis affords much finer heritability mapping, thereby reducing statistical noise in heritability estimation. To date, only one study has focused on the correlation within monozygotic twins of NAcc activation in anticipating monetary incentives (Silverman et al., 2014). The authors found a significant correlation of .38. However, it should be noted that the correlation within monozygotic twins could also be attributed to common environmental factors. Silverman and colleagues did not actually examine heritability because they did not include dizygotic twins. Therefore, it is still not clear whether these neural mechanisms of reward anticipation are heritable or not. To address this limitation, we adopted a monozygotic versus dizygotic healthy-twin design to examine the heritability of NAcc activation during the anticipation of monetary rewards using the monetary-incentive-delay (MID) task. One significant distinction of the present study is that we included both monozygotic and dizygotic twins, thus providing a more rigorous and comprehensive estimation of heritability. Moreover, we also included selfreport measures of pleasure. Previous findings support an association between motivation-related NAcc activation and self-reported pleasure (Vignapiano et al., 2016; Wacker, Dillon, \& Pizzagalli, 2009). Using a multivariate genetic model of motivation-related NAcc activation and self-reported pleasure, we explored which model was the most parsimonious and had the best fit. We hypothesized that both motivation-related NAcc activation and self-reported pleasure would exhibit significant heritability. We further hypothesized that motivationrelated NAcc activation and pleasure would share significant genetic covariance.

\section{Method}

\section{Participants}

Forty-seven pairs of same-sex dizygotic twins and 51 pairs of same-sex monozygotic twins were recruited from the Twin Registry of the Chinese Academy of Sciences Institute of Psychology (Chen et al., 2010). The zygosity of each twin was jointly determined by DNA analysis based on saliva and two zygosity questionnaires (Chen et al., 2010). Participants were excluded from the study if they (a) had a personal or family history of diagnosable mental disorders; (b) had a history of head trauma or encephalopathy; (c) had a history of substance abuse, including tobacco and alcohol; (d) had an IQ lower than 70; (e) had severe hearing or visual impairment; or (f) were ambidextrous or left handed. This information was verified by the Twin Registry, the participants themselves, and their guardians. Experimental procedures conformed to the Declaration of Helsinki, and all participants gave written informed consent. All participants completed checklists capturing experiential pleasure and hedonic traits before the brain scans were conducted. They then took the MID task inside the scanner. Each participant received $\$ 65$ as compensation plus the bonus they won in the MID task. The study was approved by the ethics committee of the Chinese Academy of Sciences Institute of Psychology.

\section{Self-report measures of pleasure}

The revised Chinese versions of the Chapman Physical Anhedonia Scale (RCPAS) and the Chapman Social Anhedonia Scale (RCSAS) were administered to measure physical and social anhedonic traits, respectively (Chan, Wang, et al., 2012). The RCPAS consists of 61 true/false items, and the RCSAS consists of 40 true/false items. These two scales have been shown to be stable and valid in measuring anhedonic traits in individuals along the schizophrenia spectrum (Chan, Gooding, et al., 2016). The Chinese version of the Temporal Experience of Pleasure Scale (TEPS) was used to measure pleasure in each participant. The TEPS is a 19-item checklist with good psychometric properties (Chan, Shi, et al., 2012).

\section{MID task}

We used an abridged imaging version of the MID task (Chan, Li, et al., 2016; the original version of the task was developed by Knutson, Westdorp, Kaiser, \& Hommer, 2000). In each trial of the task, a 250-ms cue indicating one of three different conditions was first presented at the center of the screen, followed by a blank interval between 2,000 and 2,500 ms. Then a blue 


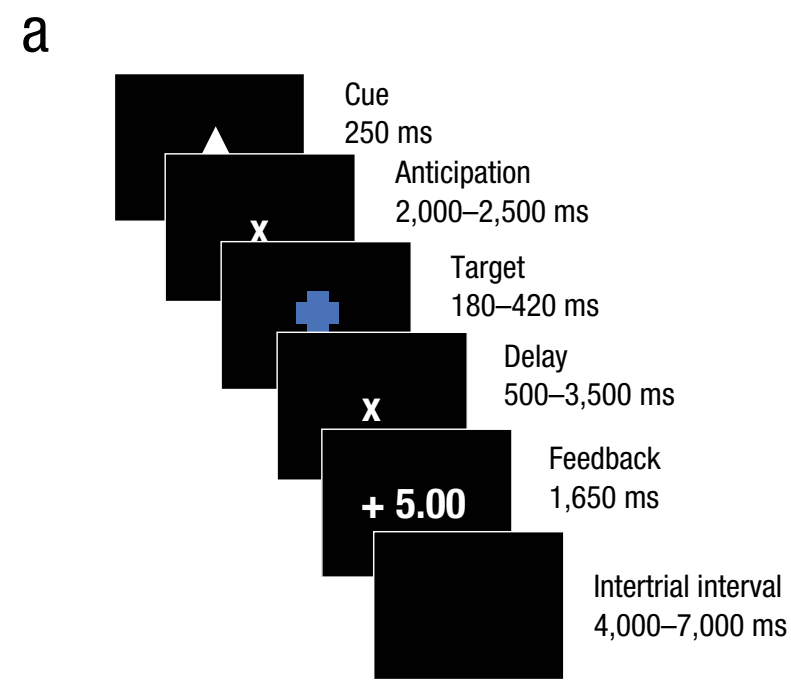

b

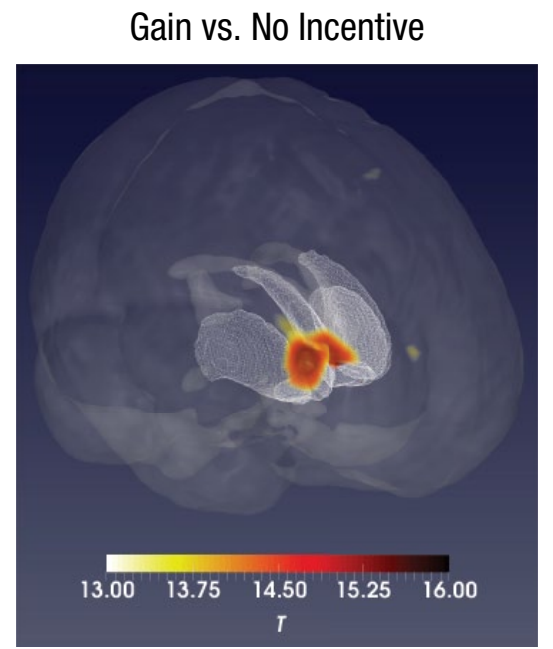

C
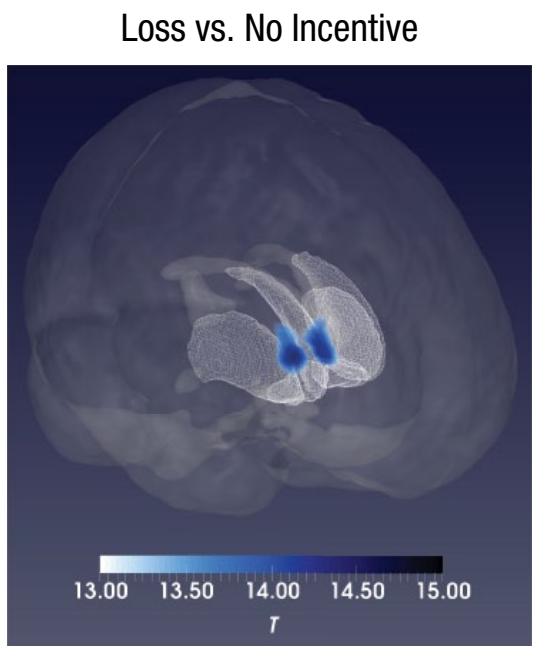

Fig. 1. Trial sequence in the monetary-incentive-delay (MID) task and brain activation during the anticipation of monetary gain and loss. In each trial of the MID task (a), a cue appeared to indicate one of three conditions (the triangle, shown here, indicated the monetary-gain condition). After a delay, participants had to hit a target. After another delay, they were told whether they won or lost points (monetary-gain and monetary-loss conditions only; no points were gained or lost in the no-incentive condition). Activations of the bilateral nucleus accumbens during the anticipatory phase is shown separately for the (b) gain-versus-no-incentive contrast and (c) loss-versus-no-incentive contrast. The color bars indicate $t$ statistics.

target with was displayed (the duration was adjusted depending on participants' responses), followed by an interval between 500 and 3,500 ms. Finally, feedback was presented for $1,650 \mathrm{~ms}$, followed by an intertrial interval between 4,000 and $7,000 \mathrm{~ms}$. The average trial lasted 12,000 ms (see Fig. 1a).

As soon as they saw the target, participants were asked to press the right button on a panel with their right thumb as quickly as possible (correct responses counted as hits). The initial duration of the target was $300 \mathrm{~ms}$ and changed according to the subsequent performance of each participant. If a target was successfully hit twice, the target duration was reduced by 20 ms. Alternatively, if a target was missed twice, $20 \mathrm{~ms}$ was added to the target duration. This strategy allowed us to control the hit rate of each participant at around $66.7 \%$. The cues that appeared at the start of each trial indicated three different conditions: A triangle indicated a monetary-gain condition, a square indicated a monetary-loss condition, and a circle indicated a no-incentive condition. Participants gained 5 points if they hit the target in the monetary-gain condition and lost 5 points if they missed 
the target in the monetary-loss condition. In the noincentive condition, participants did not gain or lose any points regardless of whether the target was hit or not. There were two runs, each containing 10 gain, 10 loss, and 10 no-incentive trials. The trials in each run were presented in a pseudorandom order. Participants practiced with an independent 30-trial run before entering the scanner and were informed that their final points gained in the scanner could be converted into cash and added to their compensation. This abridged version of the MID task has been shown to activate the NAcc effectively in healthy subclinical and clinical samples (Chan, Li, et al., 2016; Smoski, Rittenberg, \& Dichter, 2011).

\section{Brain-image acquisition}

Brain-imaging data were collected with a 32-channel head coil in a 3T Trio MRI Scanner (Siemens, Munich, Germany) at Hospital 306, Beijing. An experienced radiologist who was blind to the hypotheses of the study was responsible for data acquisition. To ascertain that each participant had no organic brain lesions, we first used a T2-weighted fluidattenuated inversion-recovery sequence (repetition time, or $\mathrm{TR}=4,000 \mathrm{~ms}$; echo time, or $\mathrm{TE}=90 \mathrm{~ms}$; field of view, or FOV $=200 \mathrm{~mm}^{2}$; slices $=19$; flip angle $=120^{\circ}$; image matrix $=256 \times 512$; voxel dimensions $=0.9 \mathrm{~mm}^{3} \times 0.4$ $\left.\mathrm{mm}^{3} \times 5 \mathrm{~mm}^{3}\right)$. Then, to acquire the functional-brainactivation data of each participant while performing the MID task, we applied a a gradient-echo echo-planar sequence $\left(\mathrm{TR}=2,000 \mathrm{~ms}, \mathrm{TE}=30 \mathrm{~ms}, \mathrm{FOV}=210 \mathrm{~mm}^{2}\right.$, slices $=32$, flip angle $=90^{\circ}$, image matrix $=64 \times 64$, voxel dimensions $=3.3 \mathrm{~mm}^{3} \times 3.3 \mathrm{~mm}^{3} \times 4 \mathrm{~mm}^{3}$, number of TRs $=184$ for each run). Finally, a high-resolution structural brain image was acquired for anatomical registration $\left(\mathrm{TR}=2,300 \mathrm{~ms}, \mathrm{TE}=3 \mathrm{~ms}, \mathrm{FOV}=256 \mathrm{~mm}^{2}\right.$, flip angle $=$ $9^{\circ}$, image matrix $=256 \times 256$, voxel dimensions $=$ $1 \mathrm{~mm}^{3} \times 1 \mathrm{~mm}^{3} \times 1 \mathrm{~mm}^{3}$ ). All participants wore earplugs during scanning. Their heads were fixed with a vacuum pillow and sponge pads to minimize head motion.

\section{Imaging-data processing}

SPM Version 12 (Wellcome Trust Centre for Neuroimaging, London, United Kingdom) was used for imagingdata processing. The functional images were realigned onto the first volume of each scanning sequence for movement correction, and slice times were then corrected. Framewise displacement was calculated for the observed head-motion parameters, three transitions and three rotations; framewise displacement is a comprehensive and reliable index of head movement (Power, Barnes, Snyder, Schlaggar, \& Petersen, 2012). Participants with head motion greater than $2 \mathrm{~mm}$ and $2^{\circ}$ and a mean framewise displacement larger than $0.25 \mathrm{~mm}$ were excluded from the final analysis, along with their twins.
Individual high-resolution brain structural images were nonlinearly registered to the Montreal Neurological Institute (MNI) template, which produced a transformation matrix. All functional brain images were normalized into a common standard atlas using this matrix. Functional images were resampled into $3 \mathrm{~mm}^{3} \times 3 \mathrm{~mm}^{3} \times$ $3 \mathrm{~mm}^{3}$ mask and spatially smoothed with a $6-\mathrm{mm}$ fullwidth, half-maximum Gaussian isotropic kernel. A 128$\mathrm{Hz}$ high-pass filter was applied to the time series of each voxel to remove low-frequency noise.

The preprocessed functional-imaging data were included in a first-level general linear model with three predictors of interest during the anticipatory phase for monetary incentives: gain, loss, and no incentive. First, the data for each participant were analyzed to provide a voxelwise $t$-statistics map for each contrast: gain versus no incentive and loss versus no incentive during the anticipatory phase. The onset of a target hit and the onset of feedback were both included as a parametric modulation to minimize their influence on the anticipation for incentives. Also, six raw head-movement parameters were included as covariates to remove motion effect. For each contrast, the $t$-statistics map of all participants was included in the general linear model with $t$ statistic as the dependent variable and framewise displacement as a covariate to further minimize the effect of head motion. To clarify whether the bilateral NAcc was activated in both monozygotic and dizygotic twins, we compared the $t$ statistics of the monozygotic and dizygotic groups using one-sample $t$ tests. The statistical significance threshold $(p)$ of the whole-brain analysis was set at less than .001, with family-wise-error correction and cluster voxel size greater than 100. Because 11 pairs of twins were excluded for excessive head movements, 44 pairs of dizygotic twins and 43 pairs of monozygotic twins were included in the final analysis.

\section{Statistical analysis}

Pearson's chi-square test was used to determine whether the gender ratios of monozygotic and dizygotic twins were different. Independent-samples $t$ tests were used to compare age and years of education between monozygotic and dizygotic twins. A univariate general linear model with gender, age, and years of education as covariates was used to compare framewise displacement and scores on the RCSAS, the RCPAS, and the TEPS between the monozygotic and dizygotic twins.

\section{Heritability brain mapping}

Voxel-by-voxel heritability brain mapping was carried out with the latest version of OpenMx software (Neale et al., 2016), the FMRIB Software Library (FSL; Jenkinson, Beckmann, Behrens, Woolrich, \& Smith, 2012), and 
in-house MATLAB (The MathWorks, Natick, MA) scripts. Because mapping the heritability of all voxels in the whole brain may increase the possibility of Type II error, we adopted the Oxford-GlaxoSmithKline-Imanova structural striatal atlas, which contains the core brain regions sensitive to dopaminergic activity and reward tasks (Tziortzi et al., 2011). The structural striatal template was first resampled into a $3 \mathrm{~mm}^{3} \times 3 \mathrm{~mm}^{3} \times$ $3 \mathrm{~mm}^{3}$ mask containing 765 voxels. The $t$ values of voxels in the striatal mask were extracted from the two contrast files (gain vs. no incentive and loss vs. no incentive) of each participant. There were no outliers more than 3 standard deviations from the mean $t$ value. Age, gender, years of education, and framewise displacement were regressed out from the extracted $t$ values to remove their possible influences on variance-component estimation (Bergen, Gardner, \& Kendler, 2007; Lenroot et al., 2009). Finally, the standardized residuals were submitted to the genetic model.

A conventional univariate $A C E$ model was used, in which $A$ denotes additive genetic effects, $C$ denotes common environmental effects, and $E$ denotes unique environmental effects. Monozygotic twins are assumed to share $100 \%$ of the additive genetic variance and common environmental variance, whereas dizygotic twins are assumed to share $50 \%$ of the additive genetic variance and $100 \%$ of the common environmental variance. The part accounted for by $A$ in the total variance was defined as the heritability estimate $\left(b^{2}\right)$ of this phenotype (Neale \& Maes, 2004). To clarify the significance of $A, C$, and $E$, we compared submodels $A E$ and $C E$ with the full $A C E$ model, and model $E$ was compared with the $A E$ and $C E$ models, respectively. If model fit significantly decreased, then the dropped factor was considered essential in the model. The model with the smallest Akaike information criterion was selected as the best-fitting model. We used the $p$ value of model comparison between the $A E$ and the $E$ model to test the significance of $h^{2}$ if the $A E$ model was detected as the best-fitting model (the full $A C E$ model failed to surpass its submodels in any voxel from the OxfordGlaxoSmithKline-Imanova structural striatal atlas; Neale \& Maes, 2004). Finally, false-discovery-rate correction with an adjusted $p$ value of less than .05 was applied to the acquired $p$ maps to adjust for multiple comparisons. Compared with methods using the full $A C E$ model in brain functional or structural studies, the method in the present study allowed us to quantify the heritability of brain activation and statistical significance in the best-fitting genetic model and to correct for multiple tests (Li et al., 2018). The cluster tool of FSL was used to identify clusters in which $h^{2}$ was significant. Masks with voxels in which the $95 \%$ confidence interval (CI) of $h^{2}$ did not contain zero were also added to the brain heritability map.

\section{Multivariate-model fit}

In the heritability brain-mapping step, we extracted $t$ values of the gain-versus-no-incentive contrast from the bilateral NAcc mask that had significant heritability. Gender, age, and years of education were then regressed from the extracted mean $t$ value, RCPAS scores, and TEPS scores to remove their possible influences on component variance estimation (Bergen et al., 2007; Lenroot et al., 2009). The framewise-displacement value was additionally regressed from the extracted mean $t$ value for head-motion correction. The heritability of self-report measures of pleasure and bilateral NAcc activation during the anticipation of monetary gain was estimated before multivariate model fitting (for details of heritability estimation, see the Heritability Brain Mapping section). The intraclass correlation coefficients of behavioral phenotypes among the monozygotic and dizygotic twins were also calculated, respectively.

Because NAcc activation during the anticipation of monetary loss failed to show significant heritability in the heritability brain-mapping step, because monozygotic twin intracorrelation was lower than dizygotic twin intracorrelation on the RCSAS, and because the effect of additive genes on social anhedonia was not significant, NAcc activation in the loss-versus-no-incentive contrast and RCSAS was not included in the multivariate genetic model. Finally, a trivariate Cholesky $A C E$ model was fitted to examine the genetic sharing between NAcc activation and pleasure (RCPAS and TEPS scores; Neale \& Maes, 2004). In addition, a trivariate variance-based $A C E$ model was also fitted to validate the Cholesky decomposition, which could induce unbiased Type I error rates (Carey, 2005). In contrast to the Cholesky $A C E$ model, which models the path coefficients, the variance-based $A C E$ model directly models the variance. The genetic covariance among the three phenotypes was estimated correspondingly in the Cholesky $A C E$ and variance-based $A C E$ models. The significance of genetic covariances was examined if the 95\% CIs included zero. In the Cholesky ACE model, the genetic contribution to the phenotype correlation between two phenotypes was estimated using the following formula (Toulopoulou et al., 2015):

$$
r_{\mathrm{ph}(\mathrm{g})}=\sqrt{\left(a_{1}^{2}\right)} \times r_{\mathrm{g}} \times \sqrt{\left(a_{2}^{2}\right)},
$$

where $a_{n}^{2}$ denotes the additive genetic component of the variance of Phenotype 1 or 2 . The genetic contribution cannot be estimated in the variance-based $A C E$ 
Table 1. Participant Demographics and Comparison Between Monozygotic (MZ) and Dizygotic (DZ) Twins

\begin{tabular}{lccccc}
\hline & & & & \multicolumn{2}{c}{$\begin{array}{c}\text { Comparison between MZ and } \\
\text { DZ twins }\end{array}$} \\
\cline { 5 - 7 } Variable & $\begin{array}{c}\text { MZ twins } \\
(n=86)\end{array}$ & $\begin{array}{c}\text { DZ twins } \\
(n=88)\end{array}$ & All $(N=174)$ & Analysis & $p$ \\
\hline Gender (male, female) & 46,40 & 46,42 & 92,82 & $\chi^{2}(1)=0.026$ & .872 \\
Age (years) & $19.98(1.89)$ & $19.8(1.76)$ & $19.89(1.82)$ & $t(172)=-0.66$ & .513 \\
Education (years) & $12.33(1.75)$ & $12.44(1.63)$ & $12.39(1.69)$ & $t(172)=0.46$ & .647 \\
Framewise displacement (mm) & $0.13(0.04)$ & $0.13(0.04)$ & $0.13(0.04)$ & $F(1,170)=0.156$ & .694 \\
RCSAS score & $11.38(5.96)$ & $7.57(4.48)$ & $9.45(5.59)$ & $F(1,170)=22.38$ & $<.001^{* * *}$ \\
RCPAS score & $22.12(8.03)$ & $18.97(7.66)$ & $20.52(7.98)$ & $F(1,170)=6.29$ & $.013^{*}$ \\
TEPS score & $4(0.52)$ & $4.15(0.58)$ & $4.07(0.59)$ & $F(1,170)=0.67$ & .574 \\
\hline
\end{tabular}

Note: For the demographic statistics, the table shows $n$ s for gender and means for all other variables. Standard deviations are given in parentheses. RCSAS = Revised Chapman Social Anhedonia Scale; RCPAS = Revised Chapman Physical Anhedonia Scale; TEPS $=$ Temporal Experience of Pleasure Scale.

${ }^{*} p<.01 .{ }^{*} p<.05$.

model because of the possible negative estimates of variance inherent in this method.

\section{Results}

\section{Demographics}

The sample included 43 pairs of same-sex monozygotic twins and 44 pairs of same-sex dizygotic twins who were matched in gender ratio, age, and years of education. In addition, their head-motion parameters were also comparable (see Table 1). Monozygotic and dizygotic twins had comparable scores on the TEPS, whereas dizygotic twins scored lower than the monozygotic twins on the RCSAS and the RCPAS.

\section{Brain activation in the gain-versus- no-incentive contrast of the MID task}

In this contrast, during the anticipatory phase, there was significant activation of the bilateral NAcc and the thalamus in all participants. Furthermore, activation of the left insula was observed in the monozygotic twins, whereas activation of the right insula was observed in the dizygotic twins (see Table 2 and Fig. 1b).

\section{Brain activation in the loss-versus-no- incentive contrast of the MID task}

For all participants, significant activation of the bilateral NAcc and the thalamus was observed in the loss-versusno-incentive contrast during the anticipatory phase. In addition, activation of the left globus pallidus and the right thalamus was observed in monozygotic twins and dizygotic twins, respectively (see Table 2 and Fig. 1b).

\section{Heritability brain mapping}

In voxel-by-voxel heritability brain mapping, two clusters were detected with significant heritability in the gain-versus-no-incentive contrast. The right cluster contained 33 voxels (voxel size $=3 \mathrm{~mm}^{3} \times 3 \mathrm{~mm}^{3} \times 3 \mathrm{~mm}^{3}$ ), whereas the left cluster contained 15 voxels. The coordinates of the peak point of the two clusters were as follows-right NAcc: $x=9, y=15, z=-3$; left NAcc: $x=-9, y=18, z=-6$ (see Fig. 2). The $b^{2}$ of each voxel within both clusters ranged from .2 to .49 , and the average $h^{2}$ was .34 (see Fig. 2). However, we did not find any significant heritability for the loss-versus-no-incentive contrast.

\section{Genetic-model fit}

The $b^{2}$ of bilateral NAcc activation during the anticipation of monetary gain was .43 $(95 \% \mathrm{CI}=[.19, .62])$, the $b^{2}$ of RCPAS scores was .61 $(95 \% \mathrm{CI}=[.39, .75])$, and the $b^{2}$ of TEPS scores was .30 (95\% CI $=[.02, .54]$; see Table 3). NAcc activation shared the same genes with RCPAS scores (Cholesky ACE model: $r_{\mathrm{g}}=-.63,95 \%$ $\mathrm{CI}=[-1,-.06]$; variance-based $A C E$ model: $r_{\mathrm{g}}=-.71,95 \%$ $\mathrm{CI}=[-1,-.11])$, whereas the genetic covariance between NAcc activation and TEPS scores was not significant (Cholesky ACE model: $r_{\mathrm{g}}=.39,95 \% \mathrm{CI}=[-.63,1]$; variance-based $A C E$ model: $\left.r_{\mathrm{g}}=.33,95 \% \mathrm{CI}=[-1,1]\right)$. In addition, scores on the RCPAS and the TEPS were influenced by some of the same genes (Cholesky $A C E$ model: $r_{\mathrm{g}}=-.81,95 \% \mathrm{CI}=[-1,-.28]$; variance-based ACE model: $\left.r_{\mathrm{g}}=-.81,95 \% \mathrm{CI}=[-1,-.41]\right)$. Additive genetic factors contributed almost $100 \%$ to the phenotypic correlation between NAcc activation and RCPAS scores, $r_{\mathrm{ph}}=-.23, r_{\mathrm{ph}(\mathrm{g})}=-.28$. Sixty-eight percent of the phenotypic correlation between RCPAS and TEPS 
Table 2. Regions With Significant Activation During the Anticipation of Monetary Incentive

\begin{tabular}{|c|c|c|c|c|c|c|}
\hline \multirow[b]{2}{*}{ Contrast and region } & \multicolumn{2}{|c|}{$\begin{array}{l}\text { Monozygotic } \\
\text { twins }(n=86)\end{array}$} & \multicolumn{2}{|c|}{$\begin{array}{c}\text { Dizygotic } \\
\text { twins }(n=88)\end{array}$} & \multicolumn{2}{|c|}{ All $(N=174)$} \\
\hline & $t$ & $\begin{array}{c}\text { Peak } \\
\text { coordinates } \\
(x, y, z)\end{array}$ & $t$ & $\begin{array}{c}\text { Peak } \\
\text { coordinates } \\
(x, y, z)\end{array}$ & $t$ & $\begin{array}{c}\text { Peak } \\
\text { coordinates } \\
(x, y, z)\end{array}$ \\
\hline \multicolumn{7}{|l|}{ Gain vs. no incentive } \\
\hline Nucleus accumbens (right) & 12.1 & $6,9,3$ & 13.41 & $-12,6,-6$ & 16.73 & $6,9,0$ \\
\hline Nucleus accumbens (left) & 11.27 & $-6,6,0$ & 12.38 & $9,9,-3$ & 16.63 & $-9,6,-3$ \\
\hline Thalamus & & & & & 14.16 & $0,-9,3$ \\
\hline Insula (left) & 10.52 & $-27,27,3$ & & & & \\
\hline Insula (right) & & & 10.26 & $33,24,3$ & & \\
\hline \multicolumn{7}{|l|}{ Loss vs. no incentive } \\
\hline Nucleus accumbens (right) & 10.7 & $0,-6,0$ & 12.22 & $-12,6,-9$ & 15.18 & $9,3,-3$ \\
\hline Nucleus accumbens (left) & 10.4 & $6,6,0$ & 11.73 & $9,3,-3$ & 15.01 & $-9,3,0$ \\
\hline Thalamus & & & & & 13.99 & $0,-6,0$ \\
\hline Thalamus (right) & & & 11.32 & $12,-3,6$ & & \\
\hline Globus pallidus (left) & 10.36 & $-9,3,0$ & & & & \\
\hline
\end{tabular}

Note: All results were family-wise-error corrected, $p<.001$, cluster voxel size $>100$. Coordinates are given in Montreal Neurological Institute space.

scores $\left(r_{\mathrm{ph}}=-.47\right)$ was attributed to additive genetic factors, $r_{\mathrm{ph}(\mathrm{g})}=-.32$ (see Figs. 3 and 4 ).

\section{Discussion}

This is the first biometric study to examine the heritability of neural substrates underlying reward processing and the shared genetic covariance of motivation-related NAcc activation and pleasure. Consistent with our hypothesis, results showed that activation at the bilateral NAcc during the anticipation of monetary gain was significantly heritable. The heritability estimate of each voxel within the bilateral NAcc, although low to moderate, was significant and ranged from .20 to .49, whereas the heritability estimate of NAcc activation as a whole was .43. Scores on the RCPAS and the TEPS also exhibited significant heritability in healthy twins, at .61 and .30 , respectively. Even with a modest sample size for $A C E$ modeling, motivation-related NAcc activation showed significant shared genetic covariation with RCPAS scores. This suggests that the significant phenotypic correlation between NAcc activation and pleasure was partially accounted for by shared genetic variation.

We found bilateral NAcc activation during the anticipatory phase for monetary incentives, which is consistent with previous findings (Knutson, Fong, Adams, Varner, \& Hommer, 2001; Knutson et al., 2000) and supports the validity of the MID task in correlating with NAcc activation in vivo. Evidence from animal studies supports the role of dopamine within the NAcc in salience attribution, an essential component of motivation and goal-directed behavior formulation (Berridge, 2003; Berridge \& Robinson, 1998). Although fMRI measures brain hemodynamics rather than neurotransmitter chemistry, previous studies have linked activation at the NAcc and the ventral striatum during rewarding tasks to local dopaminergic activity. Amphetamineinduced dopamine release at the NAcc has been associated with local hemodynamic activation in reward processing (Knutson \& Gibbs, 2007). The ventral striatal blood-oxygen-level-dependent signal has been shown to increase by optogenetic stimulations on the midbrain dopaminergic neurons of awake rats (Ferenczi et al., 2016). These data suggest that NAcc activation measured through fMRI could indirectly reflect local dopaminergic activity. In addition, polymorphisms of dopamine have been associated with ventral striatal activation in reward processing, suggesting that facets of the mesolimbic reward system may be heritable (Dreher et al., 2009; Forbes et al., 2009; Yacubian et al., 2007). Although significant monozygotic twin correlation in NAcc activation during the anticipatory period for monetary gain has previously been reported, the heritability of motivation-related NAcc was not calculated in this study without dizygotic twins (Silverman et al., 2014). Stokes and colleagues (2013) reported a significant heritability estimate of .21 for dopaminergic activities of the right ventral striatum in the resting state. However, the test-retest reliability of resting-state activation was found to be lower than NAcc activation during the anticipation of monetary incentives (Wu, 


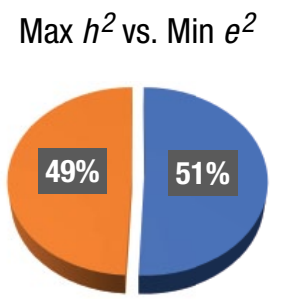

Left Peak Point

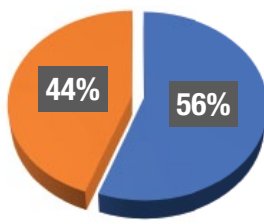

Mean $h^{2}$ vs. $e^{2}$

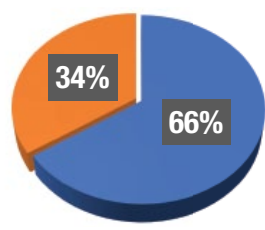

$\operatorname{Min} h^{2}$ vs. Max $e^{2}$

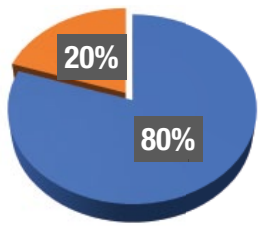

Right Peak Point

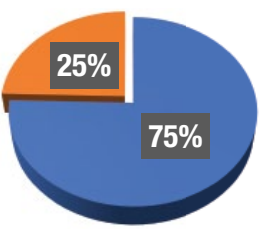

Right Putamen
Right Caudate

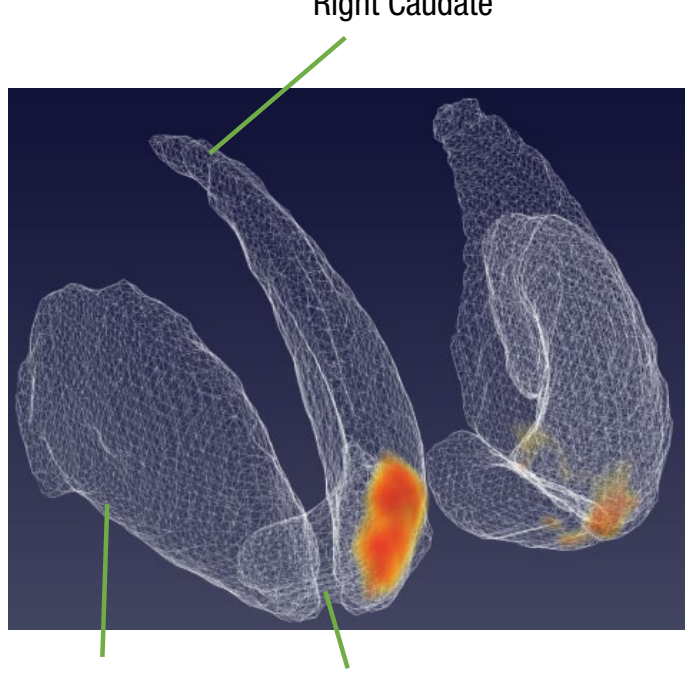

Right Ventral Striatum

Fig. 2. Voxelwise heritability mapping of brain activation. In the standard striatum atlas on the right, two clusters with significant heritability in the bilateral nucleus accumbens are highlighted. The three labeled regions were chosen arbitrarily to clarify the orientation of the brain slices shown. As reflected in the color bar, heritability estimates ranged from .20 to .49 in each voxel. The five pie charts indicate the heritability $\left(b^{2}\right)$ and unique environmental effect $\left(e^{2}\right)$ in the $A E$ model on the two clusters.

Samanez-Larkin, Katovich, \& Knutson, 2014). In the present study, we corroborated these previous findings through quantifying the genetic and environmental effects on motivation-related NAcc activation and found a heritability estimate of .40 for bilateral NAcc activation in anticipating monetary gains. The considerably larger sample size in this study, relative to previous research, further supports this finding.

Quantifying the heritability of NAcc activation in anticipating monetary incentives could facilitate the understanding of its genetic effect on reward processing and the detection of associated genetic loci. Voxelwise heritability estimation is an alternative methodology that may be more sensitive in detecting genetic effects and, thus, may supplement findings from previous ROI analyses, which are often limited by small sample sizes. Across single-nucleotide-polymorphism studies (Dreher et al., 2009; Forbes et al., 2009; Yacubian et al., 2007), twin studies (Silverman et al., 2014), and studies estimating the heritability of striatal dopaminergic activity (Stokes et al., 2013), in all of which the mean value within an ROI was used, a lateralization bias toward the right NAcc or ventral striatal activation has been reported. However, such a lateralization pattern was not observed in our

Table 3. Intraclass Correlation Coefficients for Monozygotic Twins and Dizygotic Twins and Heritability of Self-Report Measures of Pleasure and Bilateral Nucleus Accumbens Activation During the Anticipation of Monetary Gain

\begin{tabular}{|c|c|c|c|c|c|c|}
\hline Variable & $\begin{array}{l}\text { Monozygotic } \\
\text { twins }(r)\end{array}$ & $\begin{array}{l}\text { Dizygotic } \\
\text { twins }(r)\end{array}$ & $\begin{array}{l}\text { Best-fitting } \\
\text { model }^{\mathrm{a}}\end{array}$ & $b^{2}$ & $c^{2}$ & $e^{2}$ \\
\hline RCSAS score & $.381 *$ & .450 *** & $C E$ & & $.47[.29, .62]$ & $.53[.62, .38]$ \\
\hline RCPAS score & $.680 * *$ & .065 & $A E$ & $.61[.39, .75]$ & & $.39[.75, .25]$ \\
\hline TEPS score & $.486 * *$ & -.07 & $A E$ & $.30[.02, .54]$ & & $.70[.54, .46]$ \\
\hline Nucleus accumbens & $.488 * *$ & .151 & $A E$ & $.43[.19, .62]$ & & $.57[.62, .38]$ \\
\hline
\end{tabular}

Note: Values in brackets are 95\% confidence intervals. $A$ and $b^{2}=$ additive genetic effect, heritability; $C$ and $c^{2}=$ common environmental effect; $E$ and $e^{2}=$ unique environmental effect; RCSAS = Revised Chapman Social Anhedonia Scale; RCPAS = Revised Chapman Physical Anhedonia Scale; TEPS = Temporal Experience of Pleasure Scale.

${ }^{a}$ This column shows the model with the lowest Akaike information criterion.

${ }^{*} p<.01 .{ }^{* *} p<.05$. 


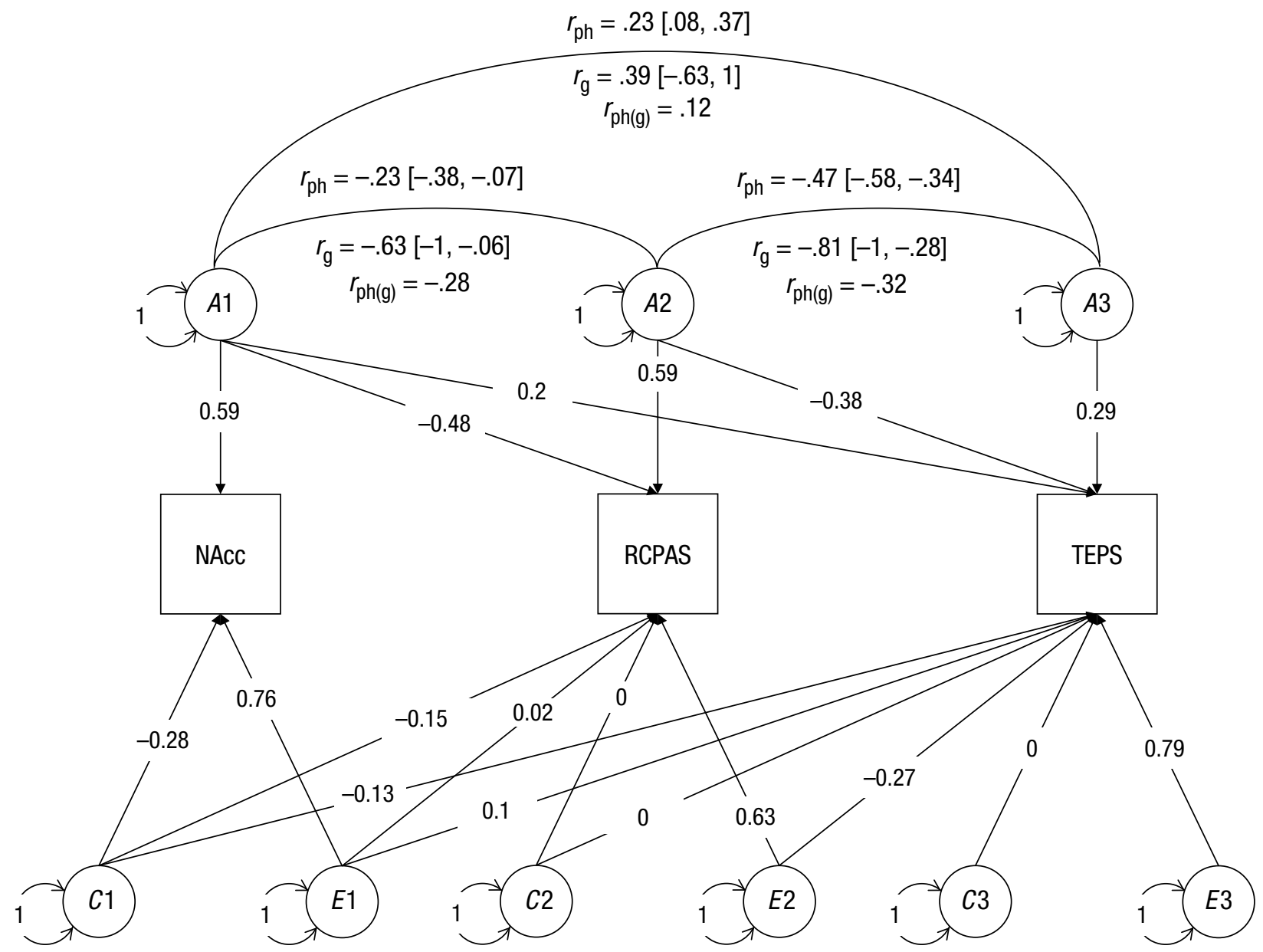

Fig. 3. Trivariate $A C E$ model with Cholesky decomposition. The three observed variables (indicated by squares) are the nucleus accumbens (NAcc), indexed using the extracted $t$ value from the gain-versus-no-incentive contrast and with the mask from the voxelwise heritability mapping of brain activation; the physical anhedonia trait, indexed using the Revised Chapman Physical Anhedonia Scale (RCPAS); and experiential pleasure, indexed using the Temporal Experience of Pleasure Scale (TEPS). $A$ indicates the additive genetic effect, $C$ indicates the common environmental effect, and $E$ indicates the unique environmental effect of each component. The correlation between phenotypes is indicated by $r_{\mathrm{ph}}$, and the genetic correlation is indicated by $r_{g}$. Circles indicate latent variables. Values on the double-headed arrows represent covariance. Values on single-headed arrows from the latent to the observed variables are standardized path coefficients.

voxelwise analysis. This is consistent with the idea that a voxelwise-heritability approach can more sensitively detect the heredity of brain activation compared with traditional ROI analysis.

It should be noted that only NAcc activation in anticipating monetary gain, rather than loss, showed significant heritability. This result is consistent with previous findings reporting a significant correlation between NAcc activation in older and younger monozygotic twins in anticipating monetary gain but not loss (Silverman et al., 2014). One possible explanation is that less individual difference exists in anticipating monetary gain than loss, even though NAcc activation appears to be sensitive to both incentive conditions. Wu and colleagues (2014) found that activation of the NAcc during the anticipation of monetary gain but not loss showed significant test-retest reliability after 2.5 years. The small or nonsignificant heritability could be attributable to the high phenotypic variance within both monozygotic and dizygotic twins resulting from limited sample size. It is notable that the studies mentioned above linked dopaminergic-gene polymorphism to ventral striatal activation in processing monetary gain but not loss, which deserves further investigation. Another noteworthy point is that the gain-versus-no-incentive and lossversus-no-incentive contrasts are conventionally taken to represent the anticipation of monetary gain and loss, respectively. However, the contrast between conditions may weaken the heritability estimates (Li et al., 2018). Hence, activation of the bilateral NAcc when one is anticipating the three monetary conditions (gain, loss, and no incentive) was also extracted and estimated for 


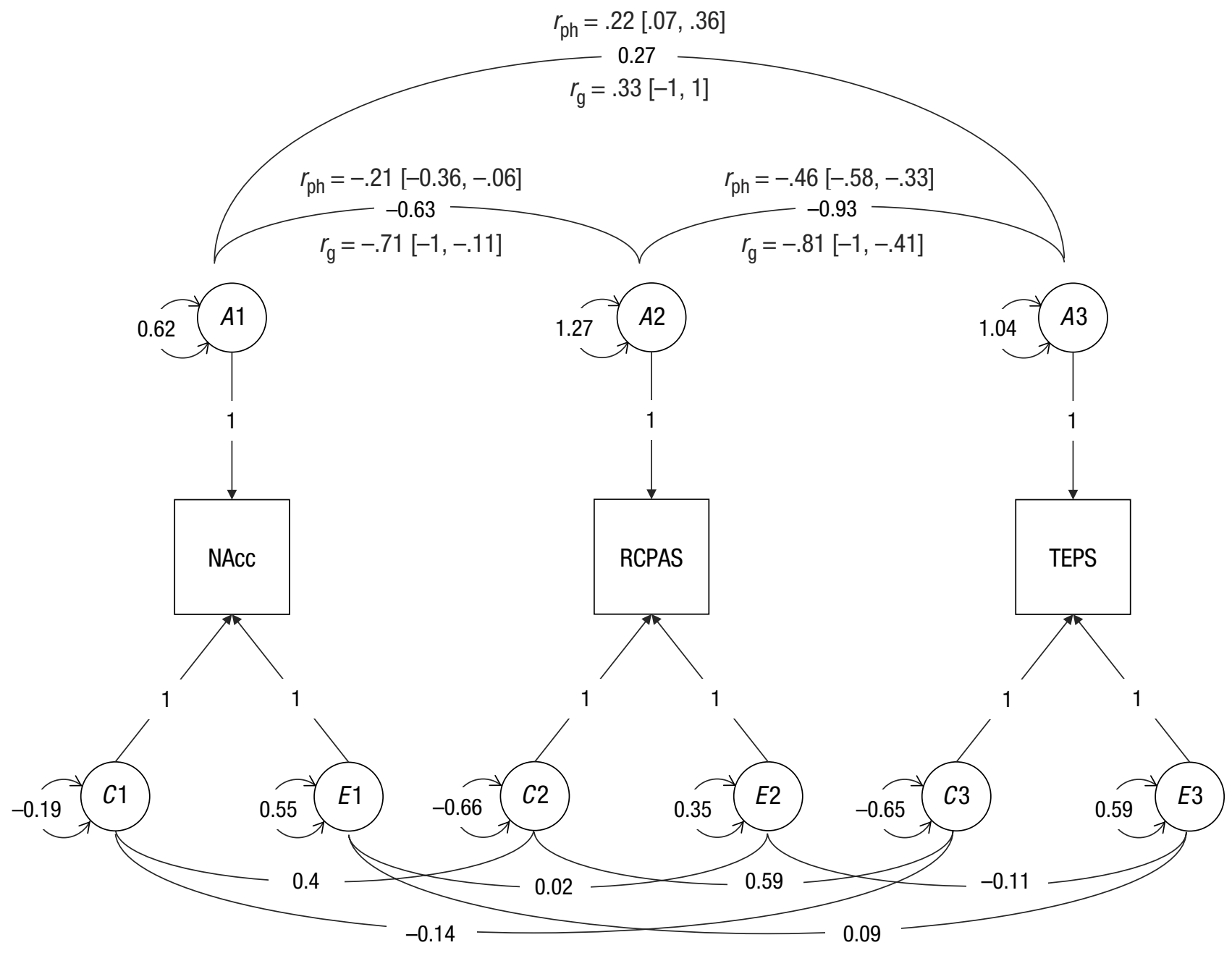

Fig. 4. Trivariate $A C E$ model with variance modeling. The three observed variables (indicated by squares) are the nucleus accumbens (NAcc), indexed using the extracted $t$ value from the gain-versus-no-incentive contrast and with the mask from the voxelwise heritability mapping of brain activation; the physical anhedonia trait, indexed using the Revised Chapman Physical Anhedonia Scale (RCPAS); and experiential pleasure, indexed using the Temporal Experience of Pleasure Scale (TEPS). $A$ indicates the additive genetic effect, $C$ indicates the common environmental effect, and $E$ indicates the unique environmental effect. The correlation between phenotypes is indicated by $r_{\mathrm{ph}}$, and the genetic correlation is indicated by $r_{g}$; the contribution to phenotypic correlation from the additive gene is indicated by $r_{\mathrm{ph}(\mathrm{g})}$. Circles indicate latent variables, and the numbers on double-headed arrows next to each latent variable indicate the variance. Values on the double-headed arrows represent covariance. Values on single-headed arrows from the latent to the observed variables are standardized path coefficients.

its heritability (see Table S1 in the Supplemental Material available online). The additive genetic effect on the anticipation of monetary loss was significant $\left(b^{2}=.36\right.$, $95 \% \mathrm{CI}=[.11, .56])$. Future research should elaborate on the experimental design with fewer contrasts between conditions.

We also investigated the heritability of pleasure. Cumulative evidence has also demonstrated a significant heritability estimate for anhedonia, ranging from .3 to .7 (Kendler \& Hewitt, 1992; Linney et al., 2003). In this study, physical anhedonia traits measured by the RCPAS also demonstrated significant heritability, whereas experiential pleasure measured by the TEPS was characterized by moderate but significant heritability. These findings are consistent with those of previous studies (Hay et al., 2001; Kendler \& Hewitt, 1992; Linney et al., 2003). In these studies, however, significant heritability has also been detected in social anhedonia, which was not found in our study. Cultural factors may be a possible influence accounting for this difference, but this requires further investigation.

The present study also highlighted the shared genetic covariance between the neural and self-reported measures of reward processing. NAcc activation during the anticipation of monetary gain exhibited significant genetic sharing with scores on the RCPAS. In addition, the phenotypic correlation between NAcc activation and self-reported pleasure was almost entirely 
attributed to genetic factors. These data suggest that motivation-related NAcc activation during the anticipation of monetary rewards may share common additive genes with pleasure, and these genes contribute significantly to the phenotypic expression. Investigating the heritability of motivation-related NAcc activation and anhedonia could help researchers identify shared regions of the genome and explore interventions for amotivation and anhedonia, both of which are associated with poor prognosis and are resistant to the currently available treatment (Kring \& Barch, 2014).

The main limitation of this study was the modest sample size, which was relatively small for statistical methods applying $A C E$ models. However, it should be noted that 196 participants with 174 valid data sets could be regarded as a medium to large sample size in task-based fMRI research. Nevertheless, we should be cautious about the generalizability of the present findings. Our findings require verification using a larger cohort including different ethnicities in future studies. On the other hand, adopting voxelwise analysis with correction for multiple comparisons could partially compensate for the relatively small sample size. One primary problem in genetic modeling lies in withingroups variation, which is sensitive to the presence of outliers; however, we detected none in this study. Furthermore, demographic variables and head movements were carefully matched between subgroups in an attempt to enhance the validity of our findings. Because the emphasis of the present study was the heritability of ventral striatal activation, which was not conclusively found during the consummatory period (Dillon et al., 2008; Radua et al., 2015), we did not examine the heritability of brain activation in receiving monetary incentives, as was done in previous studies (Forbes et al., 2009; Silverman et al., 2014; Stokes et al., 2013; Yacubian et al., 2007). Future studies could explore the heritability of brain activation during the consummatory period for various types of incentives in a large cohort.

In conclusion, our findings suggest that motivationrelated NAcc activation in anticipating monetary gain and pleasure are at least partially heritable. Importantly, motivation-related NAcc activation and pleasure exhibit significant shared genetic covariance. Future molecular studies examining shared polygenicity of these traits would further inform research in this area. Locating areas of the genome associated with expression of these traits may ultimately aid the understanding of the underlying neurobiological mechanisms of treatmentrefractory symptoms.

\section{Action Editor}

Steven W. Gangestad served as action editor for this article.

\section{Author Contributions}

Z. Li designed the study, collected and analyzed the data, and wrote the manuscript. Y. Wang and $\mathrm{C}$. Yan collected the data and commented on the first draft of the manuscript. E. F. C. Cheung, A. R. Docherty, P. C. Sham, R. E. Gur, and R. C. Gur provided significant comments on the manuscript. R. C. K. Chan conceived and designed the study, interpreted the findings, and commented critically on the manuscript. All the authors approved the final manuscript for submission.

\section{ORCID iD}

Raymond C. K. Chan (iD https://orcid.org/0000-0002-3414-450X

\section{Acknowledgments}

We thank Ting Xu, Qing Zhao, Chao Yan, Hui-jie Li, Yu-na Wang, Yan-fang Shi, Xiao-yan Cao, Weizhen Xie, Jie Chen, Xin-ying Li, Jie Zhang, and the team members of the Twin Registry of the Institute of Psychology of the Chinese Academy of Sciences for recruiting healthy twins for this study; Ke Li and Ya-wei Zeng for assisting with data collection and commenting on the first draft of the manuscript; and all the twins who took part in the study.

\section{Declaration of Conflicting Interests}

The author(s) declared that there were no conflicts of interest with respect to the authorship or the publication of this article.

\section{Funding}

This study was supported by grants from the National Key Research and Development Programme (2016YFC0906402), the National Natural Science Foundation of China (81571317), the Beijing Training Project for the Leading Talents in Science and Technology (Z151100000315020), the Beijing Municipal Commission of Science and Technology (Z161100000216138), the Strategic Priority Research Programme (B) of the Chinese Academy of Sciences (CAS; XDB02030002), the CAS Key Laboratory of Mental Health at the Institute of Psychology, and the CAS/State Administration of Foreign Experts Affairs (SAFEA) International Partnership Program for Creative Research Teams (Y2CX131003).

\section{Supplemental Material}

Additional supporting information can be found at http:// journals.sagepub.com/doi/suppl/10.1177/0956797619859340

\section{Open Practices}

Data and materials for this study have not been made publicly available, and the design and analysis plans were not preregistered.

\section{References}

Baldo, B. A., \& Kelley, A. E. (2007). Discrete neurochemical coding of distinguishable motivational processes: 
Insights from nucleus accumbens control of feeding. Psychopharmacology, 191, 439-459.

Bergen, S. E., Gardner, C. O., \& Kendler, K. S. (2007). Agerelated changes in heritability of behavioral phenotypes over adolescence and young adulthood: A meta-analysis. Twin Research and Human Genetics, 10, 423-433.

Berridge, K. C. (2003). Pleasures of the brain. Brain and Cognition, 52, 106-128.

Berridge, K. C., \& Robinson, T. E. (1998). What is the role of dopamine in reward: Hedonic impact, reward learning, or incentive salience? Brain Research Reviews, 28, 309-369.

Bossong, M. G., \& Kahn, R. S. (2016). The salience of reward. JAMA Psychiatry, 73, 777-778.

Carey, G. (2005). Cholesky problems. Behavior Genetics, 35 , 653-665.

Chan, R. C. K., Gooding, D. C., Shi, H.-S., Geng, F.-L., Xie, D.-J., Yang, Z.-Y., . . . Cheung, E. F. C. (2016). Evidence of structural invariance across three groups of Meehlian schizotypes. npj Schizophrenia, 2, Article 16016. doi:10.1038/npjschz.2016.16

Chan, R. C. K., Li, Z., Li, K., Zeng, Y.-W., Xie, W.-Z., Yan, C., . . Jin, Z. (2016). Distinct processing of social and monetary rewards in late adolescents with trait anhedonia. Neuropsychology, 30, 274-280.

Chan, R. C. K., Shi, Y.-F., Lai, M.-K., Wang, Y.-N., Wang, Y., \& Kring, A. M. (2012). The Temporal Experience of Pleasure Scale (TEPS): Exploration and confirmation of factor structure in a healthy Chinese sample. PLOS ONE, 7(4), Article e35352. doi:10.1371/journal.pone.0035352

Chan, R. C. K., Wang, Y., Yan, C., Zhao, Q., McGrath, J., Hsi, X. L., \& Stone, W. S. (2012). A study of trait anhedonia in non-clinical Chinese samples: Evidence from the Chapman Scales for Physical and Social Anhedonia. PLOS ONE, 7(4), Article e34275. doi:10.1371/journal.pone.0034275

Chen, J., Li, X., Chen, Z., Yang, X., Zhang, J., Duan, Q., \& Ge, X. (2010). Optimization of zygosity determination by questionnaire and DNA genotyping in Chinese adolescent twins. Twin Research and Human Genetics, 13, 194-200.

Dillon, D. G., Holmes, A. J., Jahn, A. L., Bogdan, R., Wald, L. L., \& Pizzagalli, D. A. (2008). Dissociation of neural regions associated with anticipatory versus consummatory phases of incentive processing. Psychophysiology, 45, 36-49.

Dreher, J. C., Kohn, P., Kolachana, B., Weinberger, D. R., \& Berman, K. F. (2009). Variation in dopamine genes influences responsivity of the human reward system. Proceedings of the National Academy of Sciences, USA, 106, 617-622.

Ferenczi, E. A., Zalocusky, K. A., Liston, C., Grosenick, L., Warden, M. R., Amatya, D., . . . Deisseroth, K. (2016). Prefrontal cortical regulation of brainwide circuit dynamics and reward-related behavior. Science, 351(6268), Article aac9698. doi:10.1126/science.aac9698

Forbes, E. E., Brown, S. M., Kimak, M., Ferrell, R. E., Manuck, S. B., \& Hariri, A. R. (2009). Genetic variation in components of dopamine neurotransmission impacts ventral striatal reactivity associated with impulsivity. Molecular Psychiatry, 14, 60-70.
Haber, S. N., \& Knutson, B. (2010). The reward circuit: Linking primate anatomy and human imaging. Neuropsychopharmacology, 35, 4-26.

Hay, D. A., Martin, N. G., Foley, D., Treloar, S. A., Kirk, K. M., \& Heath, A. C. (2001). Phenotypic and genetic analyses of a short measure of psychosis-proneness in a large-scale Australian twin study. Twin Research, 4(1), 30-40.

Iversen, L. L. (2010). Dopamine handbook. Oxford, England: Oxford University Press.

Jenkinson, M., Beckmann, C. F., Behrens, T. E., Woolrich, M. W., \& Smith, S. M. (2012). FSL. NeuroImage, 62, 782-790.

Kendler, K. S., \& Hewitt, J. H. (1992). The structure of self-report schizotypy in twins. Journal of Personality Disorders, 6, 1-17.

Knutson, B., Fong, G. W., Adams, C. M., Varner, J. L., \& Hommer, D. (2001). Dissociation of reward anticipation and outcome with event-related fMRI. NeuroReport, 12, 3683-3687.

Knutson, B., \& Gibbs, S. E. B. (2007). Linking nucleus accumbens dopamine and blood oxygenation. Psychopharmacology, 191, 813-822.

Knutson, B., Westdorp, A., Kaiser, E., \& Hommer, D. (2000). FMRI visualization of brain activity during a monetary incentive delay task. NeuroImage, 12, 20-27.

Kring, A. M., \& Barch, D. M. (2014). The motivation and pleasure dimension of negative symptoms: Neural substrates and behavioral outputs. European Neuropsychopharmacology, 24, 725-736.

Lenroot, R. K., Schmitt, J. E., Ordaz, S. J., Wallace, G. L., Neale, M. C., Lerch, J. P., . . . Giedd, J. N. (2009). Differences in genetic and environmental influences on the human cerebral cortex associated with development during childhood and adolescence. Human Brain Mapping, 30, 163-174.

Li, Z., Huang, J., Xu, T., Wang, Y., Li, K., Zeng, Y. W., . . . Chan, R. C. K. (2018). Neural mechanism and heritability of complex motor sequence and audiovisual integration: A healthy twin study. Human Brain Mapping, 39, 1438-1448.

Linney, Y. M., Murray, R. M., Peters, E. R., MacDonald, A. M., Rijsdijk, F., \& Sham, P. C. (2003). A quantitative genetic analysis of schizotypal personality traits. Psychological Medicine, 33, 803-816.

Neale, M. C., Hunter, M. D., Pritikin, J. N., Zahery, M., Brick, T. R., Kirkpatrick, R. M., . . Boker, S. M. (2016). OpenMx 2.0: Extended structural equation and statistical modeling. Psychometrika, 81, 535-549.

Neale, M. C., \& Maes, H. H. M. (2004). Methodology for genetic studies of twins and families. Dordrecht, The Netherlands: Kluwer Academic.

Power, J. D., Barnes, K. A., Snyder, A. Z., Schlaggar, B. L., \& Petersen, S. E. (2012). Spurious but systematic correlations in functional connectivity MRI networks arise from subject motion. NeuroImage, 59, 2142-2154.

Radua, J., Schmidt, A., Borgwardt, S., Heinz, A., Schlagenhauf, F., McGuire, P., \& Fusar-Poli, P. (2015). Ventral striatal activation during reward processing in psychosis: A neurofunctional meta-analysis. JAMA Psychiatry, 72, 1243-1251.

Silverman, M. H., Krueger, R. F., Iacono, W. G., Malone, S. M., Hunt, R. H., \& Thomas, K. M. (2014). Quantifying familial 
influences on brain activation during the monetary incentive delay task: An adolescent monozygotic twin study. Biological Psychology, 103, 7-14.

Smoski, M. J., Rittenberg, A., \& Dichter, G. S. (2011). Major depressive disorder is characterized by greater reward network activation to monetary than pleasant image rewards. Psychiatry Research, 194, 263-270.

Stokes, P. R., Shotbolt, P., Mehta, M. A., Turkheimer, E., Benecke, A., Copeland, C., . . . Howes, O. D. (2013). Nature or nurture? Determining the heritability of human striatal dopamine function: An [18F]-DOPA PET study. Neuropsychopharmacology, 38, 485-491.

Toulopoulou, T., van Haren, N., Zhang, X., Sham, P. C., Cherny, S. S., Campbell, D. D., . . Kahn, R. S. (2015). Reciprocal causation models of cognitive vs volumetric cerebral intermediate phenotypes for schizophrenia in a pan-European twin cohort. Molecular Psychiatry, 20, 1386-1396.

Tziortzi, A. C., Searle, G. E., Tzimopoulou, S., Salinas, C., Beaver, J. D., Jenkinson, M., . . . Gunn, R. N. (2011). Imaging dopamine receptors in humans with [11C]-(+)-PHNO: Dissection of D3 signal and anatomy. NeuroImage, 54, 264-277.

Vignapiano, A., Mucci, A., Ford, J., Montefusco, V., Plescia, G. M., Bucci, P., \& Galderisi, S. (2016). Reward anticipation and trait anhedonia: An electrophysiological investigation in subjects with schizophrenia. Clinical Neurophysiology, 127, 2149-2160.

Wacker, J., Dillon, D. G., \& Pizzagalli, D. A. (2009). The role of the nucleus accumbens and rostral anterior cingulate cortex in anhedonia: Integration of resting EEG, fMRI, and volumetric techniques. NeuroImage, 46, 327-337.

Wu, C. C., Samanez-Larkin, G. R., Katovich, K., \& Knutson, B. (2014). Affective traits link to reliable neural markers of incentive anticipation. NeuroImage, 84, 279-289.

Yacubian, J., Sommer, T., Schroeder, K., Gläscher, J., Kalisch, R., Leuenberger, B., . . . Büchel, C. (2007). Gene-gene interaction associated with neural reward sensitivity. Proceedings of the National Academy of Sciences, USA, 104, 8125-8130. 